Proceedings of Magneto-Optical Recording International Symposium '92, J. Magn. Soc. Jpn., Vol. 17, Supplement No. S1 (1993), pp. 179-182 1993 by The Magnetics Society of Japan

\title{
THERMAL CROSS-TALK ON LIGHT INTENSITY MODULATED MAGNETO-OPTICAL OVERWRITING
}

\author{
HARUKAZU MIYAMOTO, TSUYOSHI TODA, HIROSHI IDE, ATSUSHI SAITO, \\ KEIKICHI ANDOO, TOSHIO NIIHARA and MASAHIRO OJMA
}

\author{
Central Research Laboratory, Hitachi, Ltd., Kokubunji, Tokyo 185, Japan
}

\begin{abstract}
In the light intensity modulated overwriting method with exchange coupled multilayers, the erasing area surrounds the writing area because the erasing temperature is lower than the writing temperature. Therefore, the data on the adjacent track might accidentally be erased when informations are recorded on a track at a high track density. This Thermal Cross-Talk(TCT) can be effectively reduced by controlling write pulse shape and by applying a UV-resin coating on the top of the disk. By such measures, a high track density up to $1.35 \mu \mathrm{m}$ track pitch was proved feasible.

KEYWORDS:THERMAL CROSS-TALK, OVERWRITE, ERASING AREA, WRITING AREA, EXCHANGE COUPLED MULTILAYERS, TRACK PITCH, COMB-SHAPED PULSE, UV-RESIN
\end{abstract}

\section{INTRODUCTION}

Various overwriting methods have been investigated to obtain high data transfer rate for magneto-optical recording. Among them, light power modulated overwriting with exchange coupled multilayers has attracted much attention for its applicability to high-speed recording and to double-sided disks [1],[2].

In this method, the erasing temperature $T_{e}$, at which the erasing process occurs, is lower than the writing temperature $T_{w}$, at which the writing process occurs. Here, as shown in Fig.1, the data on the adjacent track might accidentally be erased when informations are recorded at a high track density. We call this Thermal Cross-Talk(TCT). Since the recording temperature in magnetooptical recording is not as high as that in phasechange recording, the TCT in magneto-optical recording is more strongly affected by the change in the ambient temperature, which make it harder to control.

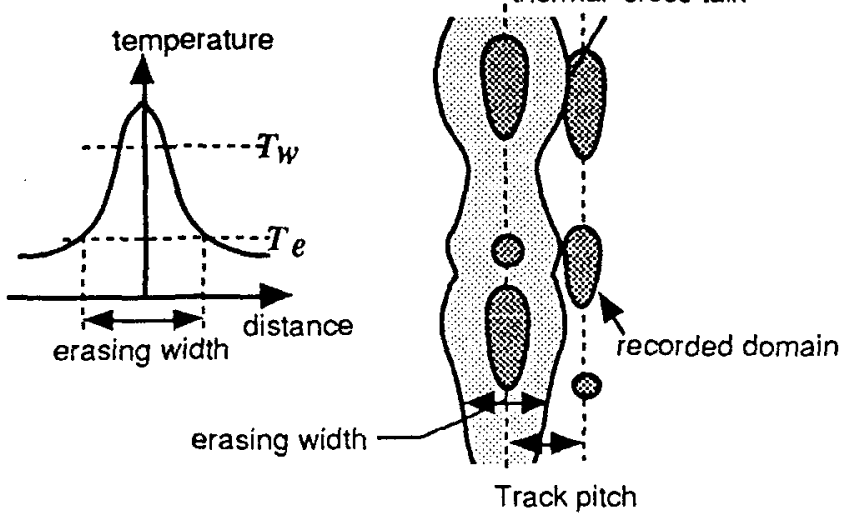

Fig.1 Temperature profile while recording and Thermal Cross-Talk(TCT)

\section{THERMAL CROSS-TALK}

\section{Thermal Structure of Recording Medium}

Figure 2 shows three typical disks with different thermal structures. On glass $2 /$ p(photopolymerization) substrates, exchange coupled multilayers(memory and writing layer) are deposited between $\mathrm{SiN}$ films(a protective layer and a dielectric layer). Here, memory and writing layers are made of $\mathrm{TbFeCo}$ and $\mathrm{TbDyFeCo}$, respectively. An interface nitride layer is inserted between them in order to control exchange coupling[3]. To study the relationship between thermal structure of the disks and TCT, three typical disks were evaluated. Disk A, Disk B and Disk $C$ are of normal, UV-resin coated and metal layer coated structures, respectively.

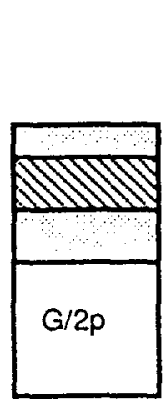

Disk A

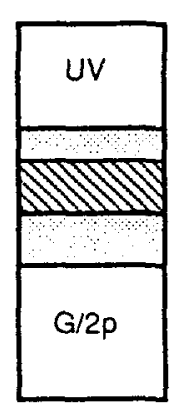

Disk B

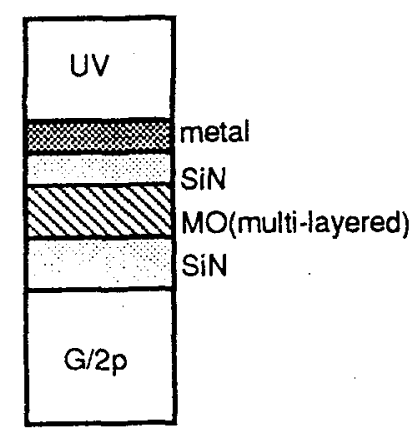

Disk C
Fig. 2 The structure of the Disks, Disk A: normal,Disk B: UV-resin coated,Disk C: UV-resin coated with metal layer.

Figure 3 shows typical overwrite characteristics on the disks in Fig.2. First, the relationship between recording laser power and output MO-signal voltage was measured. The laser power was not modulated so that stripe shaped domain should be formed on the disks. Next, 
erasing laser beam was irradiated on the stripe domains recorded with $0.6 \mu \mathrm{m}$-width and the relationship between laser power and MO-signal voltage was measured. These are the severest conditions for overwriting in pit-edge recording method. Relative erase power margins $\left(\boldsymbol{P}_{\boldsymbol{w}} \boldsymbol{s}^{-}\right.$ $\left.\boldsymbol{P}_{e c}\right) /\left(\boldsymbol{P}_{w s}+\boldsymbol{P}_{e c}\right)$ for these disks are almost the same $(\sim 28 \%)$ because three disks have the same magneto-optical multilayers with the same erasing and writing temperatures.

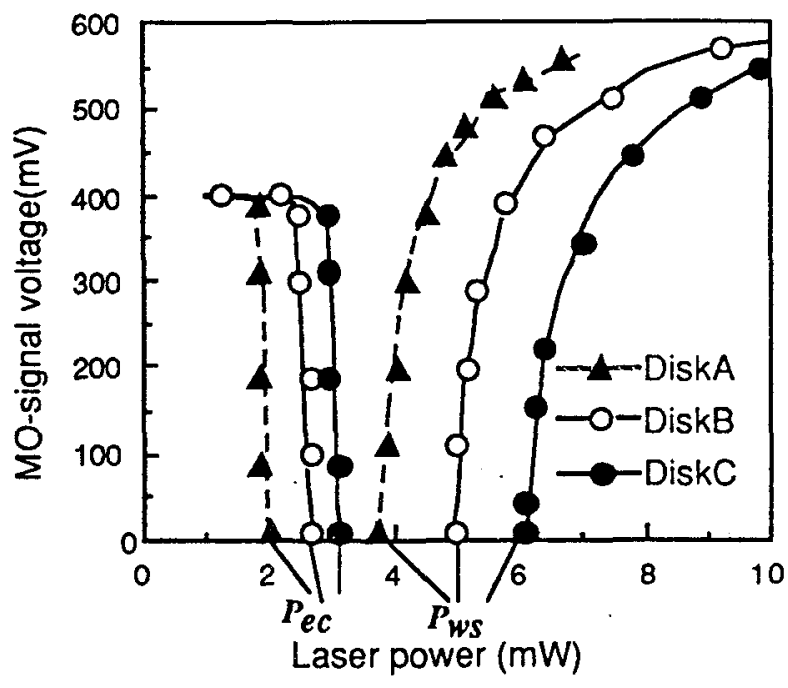

Fig.3 Overwrite characteristics

TCT was evaluated as shown in Fig.4(a). First, domains with different widths were recorded on a track. Here, we call these domains pre-recorded domains. Next, the DC(nonmodulated) laser light was irradiated on the adjacent track. Then, the minimum laser power $\boldsymbol{P}$ at which a part of the pre-recorded domains on the adjacent track can be erased was measured. In other words, $\boldsymbol{P}$ is also the maximum laser power without TCT.

The results are shown in Fig.4(b). The horizontal axis is the distance of thermal interference, which is defined to be the distance between the domain wall of the pre-recorded domains and the center of the track where the DC laser light is irradiated. Here, the power $P$ is normalized with the power $\boldsymbol{P}_{\boldsymbol{e} c}$ at which the domains on $0.6 \mu \mathrm{m}$-width on its own track can be erased completely. The power is normalized in order to compensate for the difference in sensitivity among the disks. Since $P$ is the maximum laser power without TCT and $P_{e c}$ is the minimum erasing power without erase residue, the writing and erasing power level should be set between $\boldsymbol{P}$ and $\boldsymbol{P}_{\boldsymbol{e c}}$. Therefore, the ratio $\boldsymbol{P} / \boldsymbol{P}_{\boldsymbol{e c}}$ should be large in order to ensure a good power margin. The smallest track pitch without TCT can be evaluated from the case when $\boldsymbol{P}$ equals the power for recording a $0.6 \mu \mathrm{m}$-width domain, which we call $\boldsymbol{P}_{\boldsymbol{w c}}$.

For example, in the Disk A, $\boldsymbol{P}_{w c} / \boldsymbol{P}_{e c}$ is 2.2 . Therefore, the distance of the thermal interference is $0.82 \mu \mathrm{m}$ and track pitch must be wider than $1.12 \mu \mathrm{m}$ to avoid TCT. As shown later, the change in ambient temperature and off track make this track pitch even larger.

As shown in Fig.4(b), the curve for Disk B has the steepest slope and the smallest distance of thermal interference at the same value of $\boldsymbol{P}_{\boldsymbol{w}} / \boldsymbol{P}_{\boldsymbol{e c}}$. Disk $\mathrm{C}$ has the smallest slope and the largest distance of thermal interference. This means that TCT is very strong on the thermally diffusive structure with metal layer. Therefore, on disk C, $\boldsymbol{P}_{w c} / \boldsymbol{P}_{e c}$ cannot be made large and, consequently, the erasing power margin cannot be expanded.

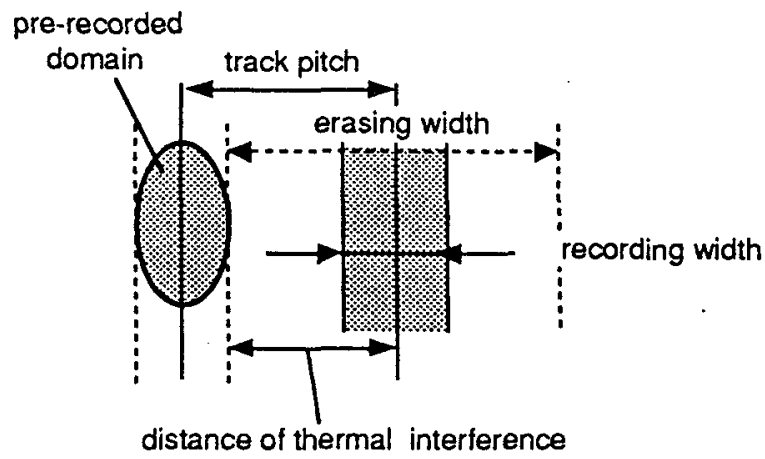

(a)

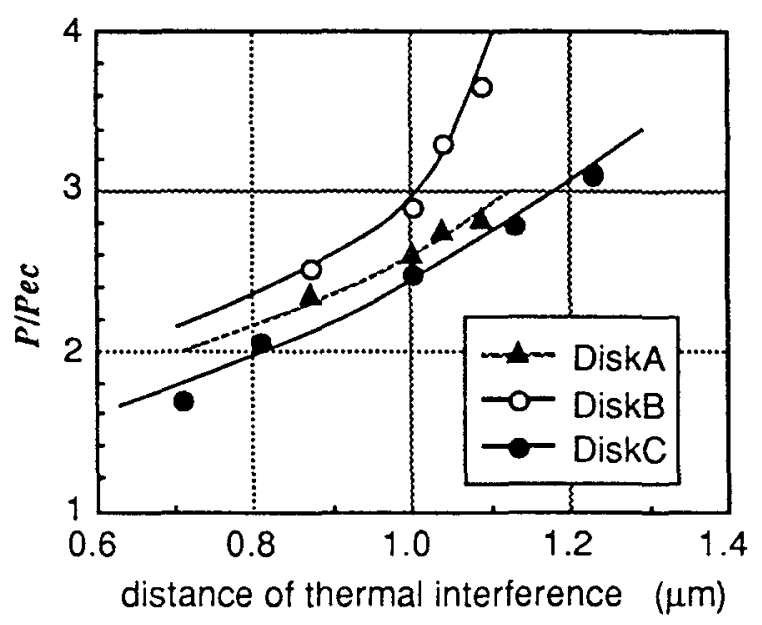

(b)

Fig.4 Thermal interference on various disk structures. (a)evaluation method for the thermal interference. (b)thermal interference.

These results can be explained from the heat flow in the disks as shown in Fig.5. In disk A, the heat flows horizontally within the magnetic layer 
which has the highest thermal conductivity in this structure. Therefore, the temperature rises much on the adjacent track. On the other hand, Disk B has UV resin on the top which has higher thermal conductivity than the air. Hence, in disk $B$, the heat can flow up into the UV resin, and heat flow within the magnetic layer is relatively small. Disk $\mathrm{C}$ has metal layer with very high thermal conductivity. Thus the heat flow within the metal layer is dominant.

We may conclude that, a structure which has thick layer with a right level of thermal conductivity on the top is the most effective in reducing TCT.

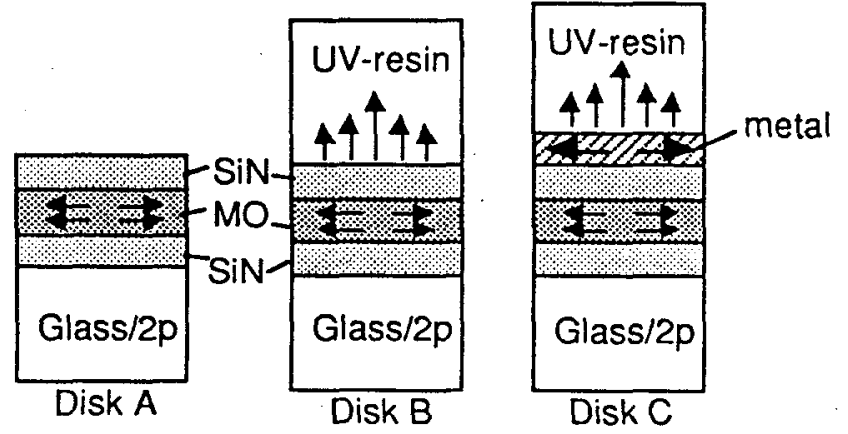

Fig. 5 Heat flow on various disk structures

\section{Control of Recording Pulse Shape}

As mentioned above, it is necessary to reduce the temperature rise on the adjacent track while recording. This means the temperature profile must be changed. It had been shown that the pulse train write compensation method is effective in reducing thermal interaction and intersymbol interference in the mark length recording method[4]. Pulse shape control like this method seems to be also effective in reducing TCT. Here, the effect of the pulse shape control was studied.

Figure 6 shows two pulse shapes, (a)rectangular and (b)comb-shaped pulses. In the comb-shaped pulses, the width of each pulse corresponds to a shift of $0.2 \mu \mathrm{m}$ in the optical spot position on the disk, which is short enough to ensure a continuous written domains.

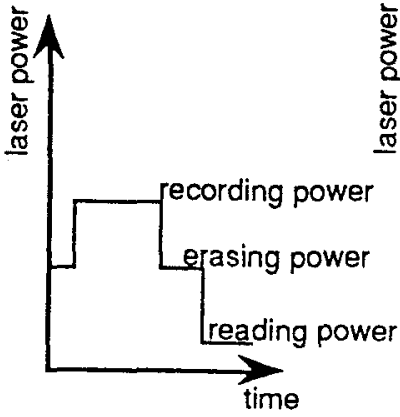

(a) rectangular pulse

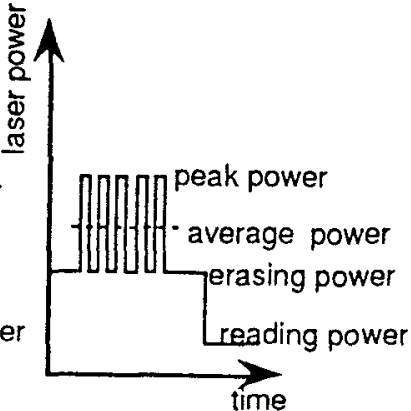

(b) comb-shaped pulses

Fig.6 Pulse shape control
Fig. 7 shows the reduction mechanism of TCT using comb-shaped pulses. When the domains are recorded on the track $A$ using comb-shaped light pulses, the average power required in recording a domain with a certain width $(\sim 0.6 \mu \mathrm{m})$ becomes smaller than when rectangular pulse is used because the temperature at the domain edge(area D) can be raised before the heat diffuses away. Therefore, only the peak power of the light defines the domain size. On the other hand, the temperature rise on the adjacent track $B$ (area $C$ ) depends on the accumulation of heat which has flowed within the magnetic layer. Therefore, the temperature rise on the adjacent track $\mathrm{B}($ area $\mathrm{C})$ does not respond to peak power but rather average power of comb-shaped pulses. Consequently, comb-shaped pulses are expected to reduce the thermal diffusion and TCT.

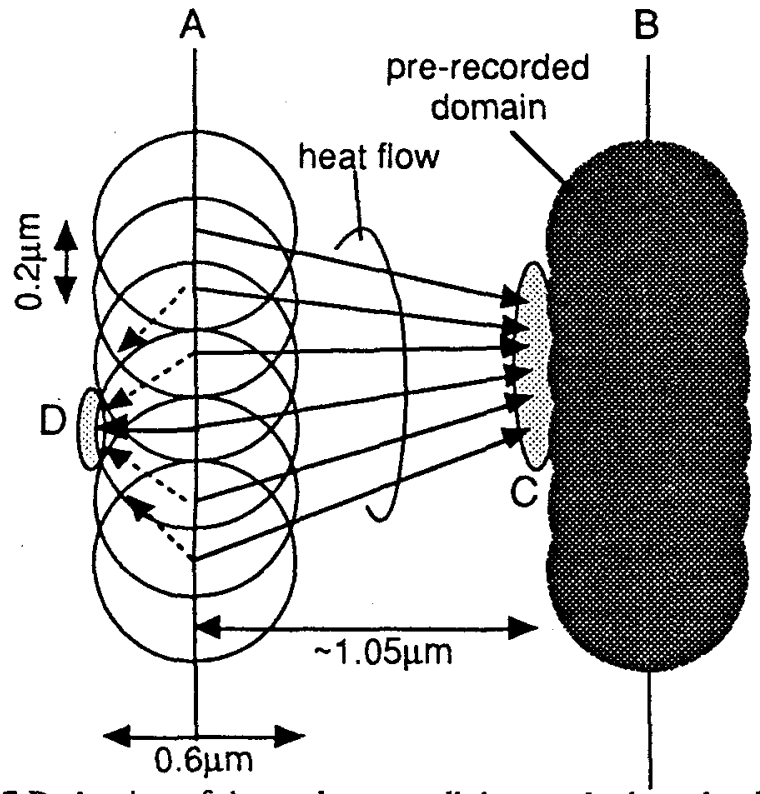

Fig.7 Reduction of thermal cross-talk by comb-shaped pulse

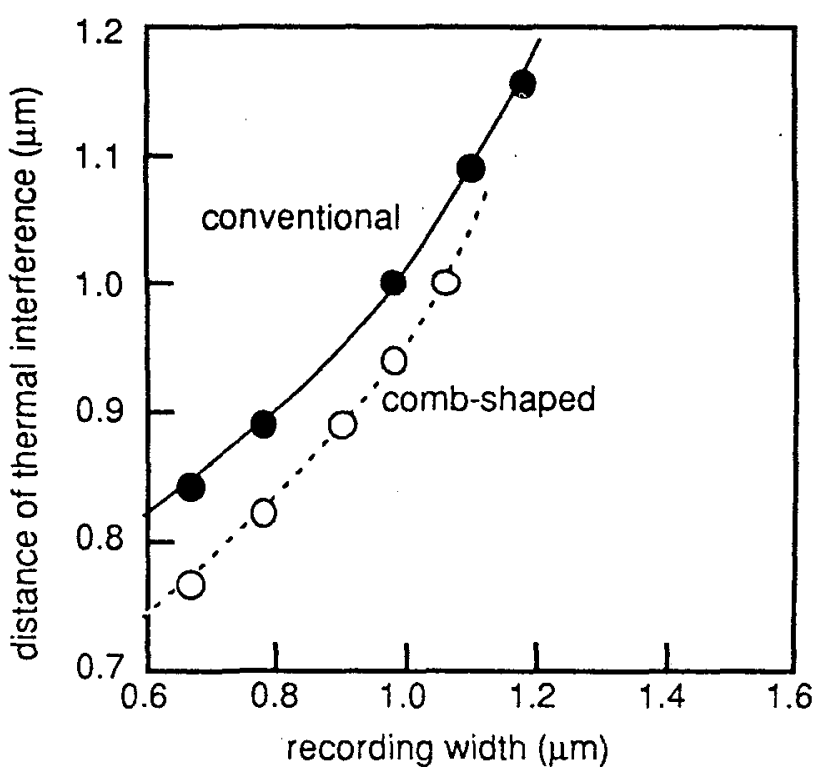

Fig.8 Thermal cross-talk with comb-shaped pulse. 
Figure 8 shows the relationship between the recording width and the distance of thermal interference when conventional DC light and comb-shaped pulse light were irradiated on disk $B$. The distances of thermal interference are measured by the same method as in Fig.4(a). Smaller erasing width was obtained by using comb-shaped pulses, which means that this pulse shape is effective in reducing TCT. When the recording width is $0.6 \mu \mathrm{m}$, the distance of thermal interference can be reduced from $0.82 \mu \mathrm{m}$ to $0.75 \mu \mathrm{m}$ using comb-shaped pulses. Consequently, the track pitch can be reduced down to $0.95 \mu \mathrm{m}$.

\section{Ambient Temperature and Off Track}

To prove the feasibility of high track density recording, the effect of the ambient temperature and the off track on to erasing width should be evaluated.

Figure 9 shows how the rise in the ambient temperature $T_{a}$ makes the erasing width larger. Figure 9 shows the temperature profiles for (a)high ambient temperature and (b)low ambient temperature. Here, the writing temperature $T_{w}$ and the erasing temperature $T_{e}$ are fixed because the temperature dependance of the magnetic characteristics does not change. The power $\boldsymbol{P}_{\boldsymbol{e c}}$ and $\boldsymbol{P}_{w c}$ are adjusted to be proportional to temperature rise $T_{e}-T_{a}$ and $T_{w}-T_{a}$, respectively. Therefore the ratio $\left(T_{w}-T_{a}\right) /\left(T_{e}-T_{a}\right)$ and $\boldsymbol{P}_{\boldsymbol{w}} / \boldsymbol{P}_{\boldsymbol{e}}$ is made larger when the ambient temperature $T_{a}$ is high.

If $T_{w}$ and $T_{e}$ are $245^{\circ} \mathrm{C}$ and $125^{\circ} \mathrm{C}$, respectively and $T_{a}$ changes from $25^{\circ} \mathrm{C}$ to $50^{\circ} \mathrm{C}$, the ratio $\left(T_{w}-T_{a}\right) /\left(T_{e}-T_{a}\right)$ increases from 2.2 to 2.6. In other words, $\boldsymbol{P}_{w c} / \boldsymbol{P}_{e c}$ increases from 2.2 to 2.6. Then, as shown in Fig.4(b), the distance of thermal interference increases to $1.0 \mu \mathrm{m}$ in the case of disk B. Therefore, the rise in the ambient temperature $T_{a}$ by $25^{\circ} \mathrm{C}$ makes the distance of thermal interference larger by $0.18 \mu \mathrm{m}$.

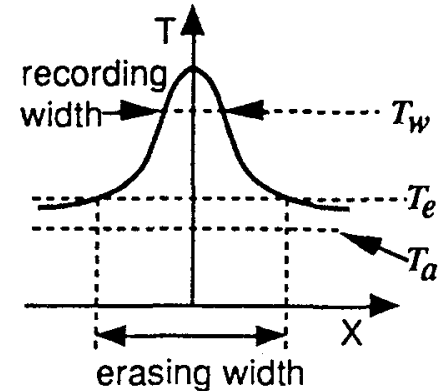

(a) High temperature $\left(50^{\circ} \mathrm{C}\right)$

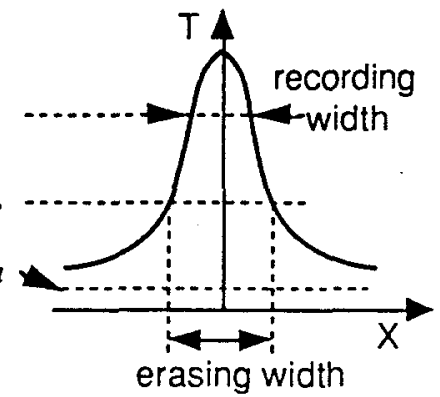

(b) Low temperaturc $\left(0^{\circ} \mathrm{C}\right)$
Fig.9 Temperature profiles
The effect of the off track is evaluated as follows. In Fig.7, if the light spot shifts to the right on the track $B$ at which the pre-recorded domain is formed and the light spot shifts to the left on the track $A$, the effective track pitch will be reduced by twice the distance of the track offset. Therefore, track pitch must be designed to be larger by that amount. For example, when the off track is $0.1 \mu \mathrm{m}$, the track pitch should be designed $0.2 \mu \mathrm{m}$ larger for that.

From the analysis discussed above, the minimum track pitch without TCT is found to be $1.33 \mu \mathrm{m}$, which is $0.95 \mu \mathrm{m}$ for the nominal condition plus $0.18 \mu \mathrm{m}$ and $0.2 \mu \mathrm{m}$ for the consideration of the ambient temperature change and the off track, respectively. This result proves the possibility of recording $1 \mathrm{~GB}$ information on each side of a $5.25^{\prime \prime}$-disk with $1.35 \mu \mathrm{m}$ track pitch.

\section{CONCLUSIONS}

Thermal cross-talk(TCT) of light intensity modulated magneto-optical overwriting, which is erasure of the domains recorded on the adjacent track, was analyzed. The comb-shaped laser pulses and use of the disk with a thick layer having moderate thermal conductivity on the top, are found to be quite effective in reducing the TCT. If the ambient temperature rise and track offset are taken in to account, $1.35 \mu \mathrm{m}$ track pitch is the minimum. This proves the possibility of recording $1 \mathrm{~GB}$ information on each side of a 5.25"-disk.

\section{REFERENCES}

[1]J.Saito et al.: Jpn.J.Appl.Phys.26(1987)Suppl. 26-4,p.155.

[2]T.Fukami et al.: J.Appl.Phys.67(1990),p.4415

[3]M.Miyamoto et al.: Proc.Moris'91 p339

[4]T.Iwanaga et al.: Proc.of Int.Symp.on Optical Memory(1991),p215 